\title{
Ciudadanía en la Red: poder y contrapoder en los medios de comunicación
}

\author{
José Ignacio CANDÓn MENA \\ Universidad de Sevilla \\ ozecai@hotmail.com
}

Recibido: 15 de marzo de 2011

Aceptado: 29 de febrero de 2012

\begin{abstract}
Resumen
Los medios de comunicación son tradicionalmente adversos a los movimientos sociales. Por ello, los movimientos tratan de incidir en el público a través de Internet pero siguen necesitando el recurso externo de los medios convencionales para incidir en la opinión pública, por lo que hacen un uso estratégico de los mismos. A través del uso de Internet los movimientos influyen en la agenda de los medios, organizando la acción colectiva de forma que no pueda ser obviada por los periodistas. También utilizan la Red para reinterpretar los mensajes mediáticos y contrarrestar los marcos simbólicos adversos al movimiento. Palabras clave: Movimientos sociales, medios de comunicación, Internet, enmarcado, agenda-setting
\end{abstract}

\section{Citizenship in the Network: power and counterpower in mass media}

\begin{abstract}
The media are traditionally adverse to social movements. Therefore, movements try to influence the public through the Internet but still need the external resources of the mainstream media to influence public opinion, so make strategic use of them. Through the use of Internet movements influence the media agenda, organizing collective action so that it can not be overlooked by journalists. They also use the Net to reinterpret the media messages and counter the adverse symbolic frames the movement.
\end{abstract}

Keywords: Social movements, media, Internet, framing, agenda-setting

Referencia normalizada: CANDÓN MENA, José Ignacio (2012): “Movimientos sociales, Internet y medios de comunicación”. Estudios sobre el mensaje periodístico, vol. 18, núm. 2 (julio-diciembre), págs.: 679-687. Madrid, Servicio de Publicaciones de la Universidad Complutense.

Sumario: 1. Introducción. Los medios de comunicación en las relaciones de poder. 2. Factores que inciden en la cobertura periodística de los movimientos. 3. Exclusión y connotación de los movimientos en los medios de comunicación. 4. Internet y la irrupción de los movimientos en la agenda mediática. 5. Internet y los marcos de interpretación de la realidad y de movilización. 6. Conclusiones. 7. Referencias bibliográficas.

\section{Introducción. Los medios de comunicación en las relaciones de poder}

La coacción y la construcción de significado son las dos formas de ejercicio del poder (CASTElls, 2009: 33). Ambas formas de poder, el poder coactivo y el poder persuasivo o discursivo, están siempre presentes, sin embargo es la segunda la forma predominante de ejercicio del poder en las sociedades modernas occidentales. Éstas se caracterizan por la existencia de formas de democracia liberal, formal o representativa y de una serie de libertades y derechos civiles y políticos -fruto a su vez de las luchas de poder históricas y de la acción de los movimientos- que limitan el uso de la violencia o la coacción en la interacciones entre Estado y sociedad. Sin embargo, el poder no desaparece en estas sociedades, en ellas se expresan relaciones asimétricas en la capacidad de influencia de determinados actores sociales que se materializan en la posesión desigual de recursos materiales, pero también simbólicos. 
Cobran importancia las nuevas desigualdades de distribución de recursos en la sociedad de la información, como la disparidad de acceso a los medios que definen los significados con los que se construye la identidad individual y colectiva. Mediante el control sobre la producción y circulación de la información los actores poderosos enmarcan la información e imponen el discurso dominante, de forma que los excluidos están privados tanto de recursos materiales como de recursos simbólicos, de su capacidad de ser sujetos (MELUCCI, 1999). El control de la información es pues el recurso clave para el ejercicio del poder, y este se ejerce sobre todo a través del sistema mundial de medios de comunicación, que junto con las instituciones médicas y de salud mental, los lenguajes para computadoras, el conocimiento del medio ambiente o el sistema político se encuentra entre los nuevos centros de poder (Op. Cit.).

Por ello, cualquiera que sea el campo de conflicto, la lucha política se encuadra siempre en el ámbito cultural y simbólico. En este contexto los movimientos sociales se esfuerzan por hacer, en primer lugar, visible la protesta y, en segundo lugar, creíble la alternativa. Pero para ello necesitaban el recurso externo de los medios de comunicación controlados por el poder. De ahí la importancia de las nuevas tecnologías de la información y la comunicación que posibilitan las mediaciones y las relaciones sociales al margen de los grandes medios que definen el discurso público. Pero el control del sistema sobre los medios de comunicación no debe entenderse en términos absolutos, como un control directo o monolítico. En la esfera de la comunicación pública se enfrentan discursos contrapuestos y tienen lugar los debates que caracterizan al sistema democrático. Sin embargo, de acuerdo con CHOMSKY y HeRMAN (1990), los medios de comunicación de masas establecen límites en el debate público produciendo un consenso tácito que limita el alcance del discurso crítico que afecta a las raíces del sistema. No es que la imagen de la realidad difundida por los medios elimine cualquier conflicto, pero sí que margina las críticas que exceden los límites del sistema $\mathrm{y}$, precisamente, los movimientos sociales son definidos por pretender la ruptura de los límites del sistema en el que actúan. De esta forma, atendiendo al tratamiento mediático de los movimientos antisistémicos podemos percibir realmente los límites impuestos por el sistema de medios de comunicación y su papel como institución de ejercicio del poder en nuestras sociedades.

Esto deriva en una gran dificultad de los movimientos para comunicarse a través de los medios convencionales, lo que provoca una actitud de rechazo por parte de los activistas que valoran de forma muy negativa el papel de los mismos, a los que identifican como parte de las estructuras de poder político y económico. Sin embargo los movimientos se enfrentan a una situación contradictoria; por una parte rechazan a los medios tradicionales y son conscientes de su limitada capacidad de incidir en ellos, por otra, perciben la necesidad de usarlos para alcanzar a un público masivo, ya que como afirma CASTELls (2009: 262), los medios no son solo un poder o el Cuarto Poder, "son mucho más importantes: son el espacio donde se crea el poder". Así "los movimientos que desean llegar a un público más amplio tienen que recurrir a los medios de comunicación para hacerlo [...] y estos no son neutrales en lo que se refiere a los símbolos que reciben y transmiten" (TARROw: 1997). 


\section{Factores que inciden en la cobertura periodística de los movimientos}

Las dificultades de los movimientos sociales para expresarse a través de los medios de comunicación tradicionales se deben a varios factores. En primer lugar, muchos de los elementos característicos de los movimientos son discordantes con los criterios, rutinas y formas de trabajo de los medios de comunicación, con la cultura profesional, la organización del trabajo y los procesos productivos de la información. Las exigencias organizativo-estructurales y las características técnico-expresivas propias de todo medio de comunicación de masas determinan la representación de la realidad ofrecida por los medios. En un contexto en el que la razón mediática contamina y subvierte la propia razón de lo político, la política mediatizada (ORTEGA, 2011) impone su lógica a la que los actores políticos están obligados a adaptarse. Una lógica que prima la personalización y el liderazgo, el poder, el reconocimiento público o la solvencia de las fuentes, la previsibilidad del acontecimiento y su cercanía, la sencillez de los temas tratados y las posiciones defendidas, etc. Estos valores-noticia o factores de noticiabilidad están alejados de la lógica y la esencia de movimientos que se caracterizan por una ausencia de liderazgo claramente definido, la espontaneidad, la profundidad de los temas tratados o la radicalidad de las críticas y propuestas defendidas.

Los medios de comunicación trabajan cómodamente cuando se trata de acudir a un acontecimiento programado en el que el líder visible de una organización formal expresa claramente su posición frente a una política concreta y cercana, sin entrar en cuestiones sistémicas o de fondo, y especialmente si esta puede enfrentarse de forma bipolar a la expresada por el partido u organización rival, lo que permite al medio justificar su pluralidad y objetividad como valores clave de la profesión periodística. De esta manera el proceso productivo de la información se simplifica, economizando costes mientras se maximaliza el público al que va dirigido el producto informativo. Así la previsibilidad de los pseudoacontecimientos ayuda a racionalizar los recursos y organizar el trabajo de los medios, el uso de fuentes solventes evita la responsabilidad sobre posibles errores cuyo coste se externaliza a las fuentes o la sencillez de los temas tratados atrae a una audiencia amplia. Sin embargo, el tema se complica cuando los medios se enfrentan a un acontecimiento imprevisible o espontáneo, protagonizado por una masa de personas a la que resulta difícil identificar, que expresa sus reivindicaciones en una amalgama de voces diversas, y que además cuestiona principios fundamentales y bien asentados de la cultura política de la sociedad, generando por tanto una conflictividad que incomoda a parte de la audiencia y limita así el mercado al que los medios masivos dirigen su producto informativo.

En definitiva, la distorsión involuntaria, derivada del carácter de la información como producto, es el primer obstáculo para una cobertura favorable de la acción de los movimientos en los medios periodísticos. Pero estas deficiencias no intencionales a la hora de tratar a los movimientos están también ligadas al segundo factor que incide en la representación periodística de los mismos y que tiene que ver con los intereses contrapuestos entre los medios de comunicación y los movimientos sociales. La mayor parte de los criterios y rutinas profesionales mencionadas pueden explicarse por la concepción de la información como un producto y la necesidad de ahorrar costes en su producción, para lo cual la previsibilidad del pseudo-acontecimiento, la bipolari- 
dad de los actores políticos, el recurso a las fuentes oficiales o solventes o el tratamiento de temas sencillos y superficiales son fundamentales. Pero más allá de los intereses económicos que afectan al proceso de producción informativa, las empresas comunicativas tienen intereses directos, tanto políticos como económicos, que pueden verse afectados por las críticas y propuestas de los movimientos de forma que la cobertura de estos se ve afectada por dichos intereses. Por tanto "los modos en que los medios cubren los movimientos y éstos son percibidos por el público se ven afectados por la estructura de la industria de la comunicación" (TARROw, 1997). No hay que olvidar que los movimientos, más allá de las protestas concretas de las que puede hacer uso cualquier actor social en un momento determinado, se caracterizan por emprender la acción colectiva de forma predominante, ya que representan reivindicaciones nuevas o no aceptadas por el sistema político y carecen de otros mecanismos para hacer valer sus propuestas. Se caracterizan por plantear cuestiones de base o sistémicas por lo cual cuestionan el sistema dominante en el que se integra la industria de la comunicación. En múltiples ocasiones son precisamente los intereses de esta industria los afectados por la acción de los movimientos, directamente o a través de los intereses de sus accionistas y anunciantes o sus aliados políticos. En resumen, la dificultad y la adversidad dan forma a la cobertura mediática de los movimientos. Estos son un actor extraño a las rutinas y formas de trabajo de los periodistas, así como un actor adverso a los intereses de la industria mediática.

\section{Exclusión y connotación de los movimientos en los medios de comunicación}

La cobertura de los movimientos se caracteriza por dos formas básicas; la primera es la exclusión o la invisibilidad, los movimientos simplemente no entran en la agenda, los acontecimientos que estos protagonizan no se convierten en noticia y éstos son obviados o al menos minimizados por los medios de comunicación. La segunda es la connotación negativa y la criminalización de los movimientos. Cuando ya no es posible obviar a los mismos se destaca la violencia por mínima que sea o se connota negativamente cualquier crítica o propuesta planteada. "En las sociedades democráticas las críticas políticas más radicales desaparecen de los medios mayoritarios porque se considera que no están a tono con la realidad del país y, por tanto, con lo que interesa a la audiencia. Sólo generando noticias (por ejemplo, manifestaciones coloristas, preferiblemente con un toque de violencia tras la intervención de la policía) pueden los radicales atravesar la barrera de los medios de comunicación. Por supuesto esto los marginaliza aún más, ya que se les identifica con la violencia y el gamberrismo, un segundo nivel de exclusión política de la opinión pública" (CASTELls, 2009: 274). Ambos fenómenos, exclusión y connotación, son bien conocidos en el estudio de las comunicaciones masivas a través de las nociones de agenda-setting y enmarcado. La incidencia de nuevos medios como Internet y el uso y apropiación de las TIC por parte de los movimientos interfiere en ambos procesos.

\section{Internet y la irrupción de los movimientos en la agenda mediática}

En cuanto a la agenda mediática y su capacidad para configurar la agenda pública, la Red permite a los movimientos difundir informaciones creando medios propios que 
potencialmente tienen una amplia difusión. Sin embargo, los medios tradicionales siguen dominando la agenda pública, tanto en sus formatos clásicos como a través de sus versiones digitales en la propia Red. Esta realidad obedece a dos factores; el primero es que Internet no ha alcanzado aún su máxima difusión y, a pesar de su amplia penetración en ciertos sectores de la sociedad, no está tan extendido como otros medios tradicionales. A pesar de ello, si atendemos a la gran aceptación del medio entre los jóvenes y a que la brecha generacional es el principal obstáculo para que el alcance de la Red se asemeje al de la radio o la televisión, podemos prever un creciente papel de Internet en la configuración de la agenda mediática. El segundo factor es la importancia de las empresas periodísticas en la generación de contenidos mediáticos. Internet ha revolucionado la economía de la distribución de contenidos, pero se ha minimizado el papel de la producción de contenidos. Así, las grandes empresas periodísticas cuentan con los recursos económicos y humanos necesarios para mantener su predominio en la producción de contenidos informativos y por tanto su control sobre la agenda pública. "Son los medios de comunicación de masas, la televisión, la radio, la prensa escrita, los que continúan realizando la mayor parte de la comunicación pública" (CARDOSO, 2008: 25). Los movimientos sociales son plenamente conscientes de esta realidad, por lo cual, manteniendo su actitud profundamente crítica con los medios tradicionales, hacen un uso estratégico de los mismos tratando de incidir en la agenda mediática para alcanzar a un público masivo e incidir así en la agenda pública. No significa que los movimientos renuncien a incidir en el público de forma directa a través de sus propios medios creados en Internet, pero a la hora de llamar la atención del público el principal uso de la Red no es directamente comunicativo sino organizativo; los movimientos utilizan la Red para organizar acciones mediáticas que atraigan la atención de los medios tradicionales.

Para organizar acciones colectivas exitosas que no puedan ser obviadas por los medios tradicionales, los movimientos deben tener éxito primero en movilizar a una masa crítica de personas, para lo que se sirven de Internet. La Red favorece los procesos de autoorganización. Reduce los costes de la acción colectiva para actores pobres en recursos como los movimientos. Se adapta a las necesidades organizativas de los movimientos dotándolos de una estructura organizativa horizontal, abierta y flexible, en la que múltiples grupos de afinidad pueden coordinarse de forma descentralizada adoptando la estructura reticular de Internet que se convierte en la infraestructura material para los colectivos informales que carecen de estructura. La forma de comunicación muchos-muchos permite la organización horizontal de grupos amplios. La interactividad del medio permite formas de participación activa y directa en el debate o la toma de decisiones. La comunicación asincrónica favorece la implicación de los activistas adaptándose a su disposición de tiempo, flexibilizando la necesidad de participar en un momento concreto y ampliando por tanto la posibilidad de involucrarse en la organización y el diseño de la acción colectiva. La deslocalización crea comunidades de interés constituidas por criterios de afinidad e independientes de la situación geográfica en las que individuos dispersos o aislados físicamente tienen la oportunidad de encontrarse y trabajar en común, generando la masa crítica suficiente de personas para impulsar y animar a la acción colectiva, coordinarla 
globalmente o llevar a cabo acciones colectivas descentralizadas. Por todo ello, la Red no es sólo un medio de comunicación sino una estupenda herramienta para la organización de los movimientos. Favoreciendo esta autoorganización de la acción colectiva la Red facilita la irrupción de los mismos en la agenda de los medios, incidiendo indirectamente en la difusión del movimiento, pero también de forma directa a través de la propia difusión del movimiento mediante los medios propios creados en Internet.

Además de organizar la acción colectiva, los movimientos adaptan sus propias formas de acción a las preferencias y rutinas de los medios de comunicación optando por acciones disruptivas no violentas o por enriquecer las acciones convencionales con elementos simbólicos o espectaculares que llamen la atención de los medios de comunicación. Pero una vez que los movimientos consiguen incidir en la agenda mediática superando la estrategia de exclusión como el primero de los filtros señalados anteriormente, éstos se enfrentan al proceso de enmarcado en el discurso mediático, ya que los medios, además de establecer la prioridad de los temas de debate público, establecen atributos a los temas tratados con lo cual las personas tienden a constituirse un punto de vista sobre los mismos.

\section{Internet y los marcos de interpretación de la realidad y de movilización}

El proceso de enmarcado en los medios incide a priori en la propia formación de los movimientos. La primera condición para que surja un movimiento social es que los actores sociales construyan su propio marco interpretativo de la realidad. Los marcos son pues los "significados compartidos o conceptos por medio de los cuales la gente tiende a definir su situación" (GAMSON, 1988). La formación del consenso (KLANDERMANS, 1988) es así el requisito previo para la movilización. Los actores sociales deben construir un marco de injusticia en el que identifican un problema y definen sus causas y responsables, un marco de acción en el que definen la estrategia adecuada para enfrentar y tratar de solucionar el problema planteado y un marco de identidad a través del cual los actores se reconocen a si mismos como un actor colectivo, como un movimiento social. Por ello, el sistema, para mantener el control, debe intervenir en las precondiciones de la acción, en la estructura motivacional, cognoscitiva y emotiva que permite que los individuos actúen, y este control se ejerce esencialmente a través de los medios de comunicación (MeluCCI, 1999). El aislamiento de los individuos que conforman el público masivo de los medios tradicionales es el escenario ideal para ejercer este control. La represión del movimiento se produce de forma preventiva en las mentes de los actores sociales a través de un discurso mediático que legitima el sistema, criminaliza la protesta y descarta las alternativas. La subjetividad de los actores es pues socialmente construida a través del intercambio y la comunicación, e Internet, como espacio comunicativo de diálogo multidireccional, juega un papel importante en la construcción de identidades e interpretaciones alternativas al discurso dominante. El flujo de informaciones, el intercambio de opiniones y experiencias o la discusión entre interpretaciones individuales de los activistas que se comunican a través de Internet son enfrentadas, contrastadas y negociadas pudiendo dar lugar a interpretaciones comunes y a propuestas comunes de acción colectiva. Internet se constituye como un nuevo espacio de socialización. Tradicionalmente, la so- 
cialización en el seno de comunidades físicas, en las que se producen las relaciones interpersonales que dan lugar a un sentimiento compartido, es el requisito indispensable para el surgimiento de una identidad colectiva, de los marcos comunes de interpretación de la realidad y de la decisión de emprender la acción colectiva. La industrialización produjo la socialización de los obreros en las fábricas o en las tabernas que permitió la configuración de una conciencia compartida -la conciencia de clase-. Los Cultural Studies han señalado la importancia de esos procesos de negociación de significados en el seno de las comunidades que comparten un mismo contexto social y generan por tanto una cultura propia que interpreta su propia realidad. Los cambios del capitalismo postindustrial, que disgregan la fuerza de trabajo en una estructura productiva que ya no se basa en las grandes industrias fordistas, sino en las redes de empresas más pequeñas, subcontratas, trabajadores autónomos, deslocalizaciones, etc., minaron las comunidades tradicionales en las que se formó la conciencia obrera. No es de extrañar por tanto que el sindicalismo tradicional siga teniendo mayor peso en los sectores de la economía industrial, en los grandes centros de producción o en la administración pública.

Hoy en Internet se crean comunidades en base a intereses compartidos, estableciéndose nuevos tipos de relaciones a través del espacio y surgiendo nuevas formas de socialización mediante la participación en foros o comunidades de interés. La Red permite establecer fuertes relaciones sociales entre personas que no comparten el mismo espacio físico pero sí un espacio virtual o ciberespacio. Howard RHEINGOLD difunde el término de comunidad virtual como "agregaciones sociales que emergen de la Red cuando un número suficiente de personas entablan discusiones públicas durante un tiempo lo suficientemente largo, con suficiente sentimiento humano para formar redes de relaciones personales en el ciberespacio". La socialización de los nuevos movimientos se produce por tanto en nuevos espacios, tanto físicos como virtuales. En resumen, el sistema mediático, a través del proceso de selección y enmarcado de la información, incide en las precondiciones para el surgimiento de la movilización social que encuentra en Internet una vía alternativa para la construcción de interpretaciones antagónicas de la realidad. Pero también cuando el movimiento actúa, una vez que ha superado esa fase previa de construcción de un marco de movilización y pasa del estado latente al estado de actividad, sigue estando afectado por el poder de enmarcado del sistema de medios. Los movimientos que consiguen organizarse y emprender la acción colectiva pueden llegar a superar la estrategia de exclusión mediática irrumpiendo en la agenda de los medios, pero los medios aún pueden retomar el control a través del enmarcado del movimiento, desplegando una retórica reactiva (HIRSCHMAN, 1991) que destaca el riesgo, la futilidad y los efectos perversos de la acción colectiva, aislando al movimiento de su base social. Los movimientos tratan de contrarrestar este poder de los medios de dar forma a las percepciones del público atrayéndolo hacia sus medios propios en Internet. Pero también la información difundida en los medios masivos puede impulsar a la acción incluso cuando esté fuertemente (y negativamente) connotada. El mensaje puede ser descodificado desde un marco de oposición, que interpreta el mismo a partir de otro marco de referencia, con una visión contraria del mundo (HaLl, 1973). De acuerdo con la tradición de los Cultural Studies o los estu- 
dios semióticos, la recepción de los mensajes mediáticos se produce en un proceso de reinterpretación que puede ser contrario a las intenciones del emisor. Así podemos afirmar que superado el filtro de la agenda, los movimientos ya han conseguido un objetivo importante y en ciertos casos puede ser válida la afirmación de que lo importante es que se hable de uno, aunque sea mal.

En este sentido es necesario atender al ecosistema mediático actual, en el que conviven los medios tradicionales y los nuevos medios como Internet. La Red contribuye al despliegue y consolidación de redes de relaciones sociales en las que se reinterpretan los mensajes mediáticos. La realidad es filtrada por los medios convencionales, pero del mismo modo los mensajes mediáticos son filtrados por las redes sociales en Internet. Los comentarios en los foros y páginas de información on-line, la redistribución de noticias a través de listas y correos electrónicos, la difusión de interpretaciones y opiniones en la blogosfera, etc., pueden alterar el marco interpretativo de la realidad difundido por los medios. El fenómeno de los agregadores de noticias, como meneame.net, son un buen ejemplo de los procesos de reelaboración de la información en los nuevos medios. En primer lugar, se reelabora la agenda de los medios a través del envió de informaciones de otros sitios web -que pueden o no ser versiones digitales de los medios tradicionales- y de la selección de las mismas a través de la votación de los usuarios. De esta forma se crea una agenda propia socialmente construida con criterios que pueden diferir de los valores y rutinas periodísticas de los medios convencionales. En segundo lugar, se enmarcan e interpretan las informaciones publicadas a través de los comentarios de los usuarios a las noticias, que pueden ser a su vez votados por otros usuarios. De esta forma los comentarios de las noticias pueden reinterpretar la información publicada dando al acontecimiento cubierto una connotación opuesta a la del medio que publica la noticia. Este proceso de selección y comentarios se da también en otras herramientas de Internet como las Redes Sociales en Internet (RSI). En resumen, a través de la Red se reelabora socialmente tanto la agenda como el enmarcado mediático de la realidad, lo que supone una oportunidad para los movimientos sociales que limitan así su dependencia respecto al sistema de medios de comunicación.

\section{Conclusiones}

Internet abre nuevas oportunidades a los movimientos sociales que tratan de incidir en la opinión pública. La Red limita la dependencia de los movimientos respecto al sistema de medios de comunicación convencional, que por sus propias rutinas productivas y por sus intereses económicos como empresas capitalistas son tradicionalmente adversos a los movimientos sociales que cuestionan las raíces del sistema en el que éstas actúan. A través de Internet los movimientos irrumpen en la agenda de los medios tradicionales organizando la acción colectiva de forma que ésta no pueda ser ignorada por los medios convencionales. El papel de la Red es en este sentido más organizativo que directamente comunicativo, aunque a través de medios propios en la Red los movimientos tratan también de establecer sus propias agendas alternativas. Por otra parte, los movimientos crean y difunden sus propios marcos de interpretación de la realidad a través de la Red y en ésta se reinterpretan los contenidos mediáticos. A 
pesar de todo, los medios tradicionales siguen teniendo un papel predominante a la hora de establecer los temas de interés de la opinión pública y los marcos de interpretación social de la realidad, pero este poder es cuestionado a medida que Internet se expande como medio de comunicación (superando la brecha generacional a través de la renovación de las audiencias) y se acumulan las experiencias del uso social de la tecnología para la protesta. La Red no sustituirá al sistema tradicional de los medios de comunicación, pero lo complementa e incide en las propias agendas y marcos interpretativos de los medios convencionales. Los movimientos sociales, conscientes de ello, usan la Red para difundir sus puntos de vista y para influir en los propios medios de comunicación.

\section{Referencias Bibliográficas}

CARDOSO, Gustavo (2008): Los Medios de Comunicación en la Sociedad Red: Filtros, Escaparates y Noticias. Barcelona, UOC.

CASTELLS, Manuel (2009): Comunicación y poder. Madrid, Alianza Editorial.

CHOMSKY, Noam y HERMAN, Edward S. (1990): Los guardianes de la libertad. Barcelona, Crítica.

GAMSON, William (1988): Political Discourse and Collective Action, en KLANDERMANS, Bert; KRIESI, Hanspeter y SIDNEY Tarrow (compiladores): International Social Movement Research: From Structure to Action. Greenwhich, Connecticut: JAI Press.

HALL, Stuart (1973): Encoding and decoding in the television discourse. Birmingham, Centre for Contemporary Cultural Studies.

HIRSCHMAN, Albert (1991): Retóricas de la intransigencia. México, Fondo de Cultura Económica.

KLANDERMANS, Bert (1988): The Formation and Mobilization of Consensus, en KLANDERMANS B., KRIESI H. y TARROW S. (eds), International Social Movements Research. Supplement to Research in Social Movements, Conflicts, and Change. vol,1. Greenwich, Connecticut, JAI Press.

MELUCCI, Alberto (1999): Acción Colectiva, Vida Cotidiana y Democracia. México, El Colegio de México.

ORTEGA, Félix (2011): La politica mediatizada. Madrid, Alianza.

RHEINGOLD, Howard. (1996): La comunidad virtual. Una sociedad sin fronteras. Barcelona, Gedisa.

TARROW, Sidney (1997): El poder en Movimiento. Madrid, Alianza. 